\title{
Relationship between salivary/pancreatic amylase and body mass index: a systems biology approach
}

Amélie Bonnefond $^{1 *+}$, Loïc Yengo ${ }^{1,2+}$, Aurélie Dechaume ${ }^{1}$, Mickaël Canouil ${ }^{1}$, Maxime Castelain ${ }^{1}$, Estelle Roger ${ }^{1}$, Frédéric Allegaert ${ }^{1}$, Robert Caiazzo ${ }^{3,4}$, Violeta Raverdy ${ }^{3,4}$, Marie Pigeyre ${ }^{3,4}$, Abdelilah Arredouani ${ }^{5}$, Jean-Michel Borys ${ }^{6}$, Claire Lévy-Marchal ${ }^{7}$, Jacques Weill ${ }^{8}$, Ronan Roussel ${ }^{9,10,11}$, Beverley Balkau ${ }^{12}$, Michel Marre ${ }^{9,10,11}$, François Pattou ${ }^{3,4+}$, Thierry Brousseau ${ }^{13 \dagger}$ and Philippe Froguel ${ }^{1,14^{*}}$

\begin{abstract}
Background: Salivary (AMY1) and pancreatic (AMY2) amylases hydrolyze starch. Copy number of AMY1A (encoding AMY1) was reported to be higher in populations with a high-starch diet and reduced in obese people. These results based on quantitative PCR have been challenged recently. We aimed to re-assess the relationship between amylase and adiposity using a systems biology approach.

Methods: We assessed the association between plasma enzymatic activity of AMY1 or AMY2, and several metabolic traits in almost 4000 French individuals from D.E.S.I.R. longitudinal study. The effect of the number of copies of AMY1A (encoding AMY1) or AMY2A (encoding AMY2) measured through droplet digital PCR was then analyzed on the same parameters in the same study. A Mendelian randomization analysis was also performed. We subsequently assessed the association between AMY1A copy number and obesity risk in two case-control studies (5000 samples in total). Finally, we assessed the association between body mass index (BMI)-related plasma metabolites and AMY1 or AMY2 activity.
\end{abstract}

Results: We evidenced strong associations between AMY1 or AMY2 activity and lower BMI. However, we found a modest contribution of AMY1A copy number to lower BMI. Mendelian randomization identified a causal negative effect of BMI on AMY1 and AMY2 activities. Yet, we also found a significant negative contribution of AMY1 activity at baseline to the change in BMI during the 9-year follow-up, and a significant contribution of AMY1A copy number to lower obesity risk in children, suggesting a bidirectional relationship between AMY1 activity and adiposity. Metabonomics identified a BMI-independent association between AMY1 activity and lactate, a product of complex carbohydrate fermentation.

Conclusions: These findings provide new insights into the involvement of amylase in adiposity and starch metabolism.

Keywords: AMY1AVAMY2A, Body mass index, Copy number variant, Mendelian randomization, Metabonomics, Obesity, Salivary/Pancreatic amylase, Starch

\section{Background}

Amylase is responsible for starch hydrolysis, initiating carbohydrate digestion in the oral cavity and later in the gut. In humans, approximately half of the amylase activity found in serum is produced by the salivary glands and the remaining part by the exocrine pancreas

\footnotetext{
* Correspondence: amelie.bonnefond@inserm.fr; froguel@good.ibl.fr ${ }^{\dagger}$ Equal contributors

'University of Lille, CNRS, Institut Pasteur de Lille, UMR 8199 - EGID, Lille 59000, France

Full list of author information is available at the end of the article
}

[1] from different genes located in the same complex chromosome 1 locus.

A well-known multi-allelic copy number variant at salivary amylase gene (AMY1A; diploid copy number ranging from one to roughly 20 ) evolved as an adaptation to dietary habits [2]. Populations with high starch consumption carry larger number of copies than others that have maintained an ancestral pre-agricultural way of life [2]. Previously, we reported that $A M Y 1 A$ copy number estimated by quantitative RT-PCR (qPCR) is associated with body mass index (BMI) in North European and 
South Asian adult populations [3]. It provided a putative genetic link between complex carbohydrate metabolism in the gut and obesity. This association was replicated in early-onset obese females from Finland [4] and in prepubertal boys in Italy [5], and an association with insulin resistance was reported in adult Korean men [6], where $A M Y 1 A$ copy number was also estimated by qPCR. On the other hand, using digital PCR, two studies failed to reproduce these findings $[7,8]$. Usher et al. [7] suggested that the discrepancy with the previously reported observations likely comes from their higher-resolution approaches for both molecular and computational analyses. Recently, however, using digital PCR, we have found that, in Mexican children with high-starch diet, high number of $A M Y 1 A$ copies significantly protects against obesity in this population [9]. Finally, a study that used fiber-FISH suggested a role for copy number of pancreatic amylase genes ( $A M Y 2 A$ and $A M Y 2 B$ ) in the observed functional associations [10].

This debate is important for several reasons. First, chromosome structural variants are increasingly recognized to highly contribute to disease development [11], and thus the correct genotyping of multi-allelic copy number variant is mandatory [12]. Second, it was shown that non-obese adults with high salivary amylase activity (and putatively high $A M Y 1 A$ copy number) present with improved glucose tolerance following liquid starch ingestion [13]. Furthermore, high serum amylase activity was shown to be associated with decreased risk of metabolic syndrome and type 2 diabetes in a Japanese asymptomatic population [14]. Finally, in more than 100 different strains of mice fed a high-fat, high-sucrose diet, the Amy1 locus was reported to be significantly associated with weight gain variation and with an enrichment of obesity-associated bacteria of gut microbiota [15]. Therefore, it is crucial to robustly determine if amylase activities (and amylase gene copy number) impact energy and glucose homeostasis.

In the present study, we employed a systems biology approach, using genetics, protein activity and metabonomics analyses, to decipher the putative interaction between amylase genes and adiposity in human population. We first assessed the association between plasma enzymatic activity of salivary (AMY1) or pancreatic (AMY2) amylase, and several metabolic traits, including BMI. We then analyzed the effect of $A M Y 1 A$ or $A M Y 2 A$ copy number on the same parameters. A Mendelian randomization analysis was subsequently performed to assess causality effects explaining the complex relationship between BMI and AMY1 or AMY2 plasma enzymatic activity, and actually suggested a bidirectional causal negative effect in the relationship between BMI and AMY1 plasma enzymatic activity. We subsequently confirmed an association between $A M Y 1 A$ copy number and reduced obesity risk in children. Finally, we assessed the association between BMI-related plasma metabolites and AMY1 or AMY2 plasma enzymatic activity.

\section{Methods \\ Study participants \\ D.E.S.I.R}

D.E.S.I.R. is a 9-year longitudinal study in a French general population, fully described elsewhere [16]. A total of 4834 unrelated individuals who were successfully genotyped through iSelect Metabochip DNA microarrays (Illumina, San Diego, CA, USA) was included in the present study. $A M Y 1 A$ copy number and $A M Y 2 A$ copy number were successfully genotyped in 3607 and 3657 participants, respectively. At baseline, we had access to AMY1 plasma enzymatic activity for 3744 participants. Among them, we had access to AMY1 plasma enzymatic activity after 9 years of follow-up for 679 individuals, to BMI after 9 years of followup for 2796 individuals, and to the levels of BMI-associated plasma metabolites at baseline for 718 individuals. Moreover, we had access to AMY2 plasma enzymatic activity at baseline for 3980 participants. Among them, we had access to AMY2 plasma enzymatic activity after 9 years of followup for 705 individuals, to BMI after 9 years of follow-up for 2970 individuals, and to the levels of BMI-associated plasma metabolites at baseline for 718 individuals. Additional file 1 recapitulates all these numbers. Non-diabetic participants did not use glucose lowering medication, and presented with fasting plasma glucose less than $7 \mathrm{mmol} / \mathrm{L}$ and glycated hemoglobin A1c less than 6.5\% [17].

\section{The Biological Atlas of Severe Obesity study (ABOS)}

ABOS is a cohort study (ClinicalGov NCT01129297) from the University Hospital of Lille, France, fully described elsewhere [18]. In the present study, we measured plasma enzymatic activity of AMY1 and AMY2 in 488 participants who were also genotyped through Metabochip DNA microarrays (Illumina).

\section{Obesity case-control studies}

Clinical characteristics of study participants are shown in Additional file 2. The first case-control study included 2220 normal-weight adults (with a $\mathrm{BMI}<25 \mathrm{~kg} / \mathrm{m}^{2}$ ) and 1179 adults presenting with obesity (with a $B M I \geq 30 \mathrm{~kg} / \mathrm{m}^{2}$ ). These adults were from D.E.S.I.R. or were recruited either by the CNRS UMR8199 (Lille, France), by the Department of Nutrition of Hotel-Dieu Hospital (Paris, France), or by the Centre d'Etude du Polymorphisme Humain $(\mathrm{CEPH}$, Saint-Louis hospital, Paris, France). The second case-control study included 712 normal-weight children or adolescents (with a BMI-for-age $<85$ th percentile) and 785 children or adolescents presenting with obesity (with a BMI-for-age $\geq$ 99th percentile). These children or adolescents were from the French Haguenau regional cohort study [19] or from the French Fleurbaix-Laventie Ville Santé study [20], or they were recruited by the CNRS UMR8199 (Lille, France). 
Estimation of AMY1A and AMY2A copy number

Copy number of $A M Y 1 A$ and $A M Y 2 A$ was estimated using the QX200 droplet digital PCR (ddPCR) system (Bio-Rad Laboratories, Hercules, CA, USA), following the manufacturer's recommendations. Concentration of DNA samples was measured using the Qubit ds DNA Assay HS kit (Life Technologies, Carlsbad, CA, USA). Dilutions were performed with 20× GE Sample Loading Reagent (Fluidigm, South San Francisco, CA, USA). Each $40 \mu \mathrm{L}$ reaction included $11 \mu \mathrm{L}$ ddPCR SuperMix for Probes no dUTP (Bio-Rad), 24 ng DNA (for $A M Y 1 A$ copy number estimation) or $32 \mathrm{ng}$ DNA (for $A M Y 2 A$ copy number estimation), $1.1 \mu \mathrm{L}$ of TaqMan assay targeting $A M Y 1 A$ or $A M Y 2 A$ (Hs07226362_cn or Hs04204136_cn, respectively; Life Technologies), $1.1 \mu \mathrm{L}$ of TaqMan assay targeting the reference RNase $P$ assay (Human RNase P \#4403328; Life Technologies), and 0.5 U HindIII (High Fidelity; New England Biolabs, Ipswich, MA, USA). Of note, both $A M Y 1 A$ and $A M Y 2 A$ target assays utilized FAM-labeled probes, while RNase $P$ assay was labeled in VIC. Enzymatic digestion was done for 5 minutes at $20{ }^{\circ} \mathrm{C}$. Subsequently, the reaction was emulsified with Droplet Generator Oil (Bio-Rad) using the QX200 Droplet Generator (Bio-Rad), following the manufacturer's instructions. The droplets were then transferred to a 96-well reaction plate (Eppendorf) and PCR amplification was performed using a Veriti Thermal Cycler (Life Technologies). After amplification, droplets were read using a QX200 Droplet Reader (Bio-Rad). Fluorescence data were analyzed using QuantaSoft software (version 1.7.4, Bio-Rad). Only samples with at least 7000 droplets were kept for further analyses.

\section{Measurement of plasma enzymatic activities of salivary (AMY1) and pancreatic (AMY2) amylases}

Plasma enzymatic activities of total amylase and AMY2 were estimated by an enzymatic colorimetric assay with an autoanalyzer (CoBAS Icobas 8000 modular analyzer series; kits \#AMY-P-20766623322 and \#AMYL2-03183742122; Hoffman-La Roche, Basel, Switzerland). The plasma enzymatic activity of AMY1 was calculated by subtracting the activity of AMY2 from the activity of total amylase. Normal ranges of the plasma enzymatic activities of AMY2 and total amylase were 13-53 U/L and 29-99 U/L, respectively. Only individuals presenting with these normal ranges were analyzed.

\section{Measurement of plasma metabolites}

Fasting plasma samples were processed by the Metabolon (Durham, NC, USA) platform using gas chromatography mass spectrometry and liquid chromatography-tandem mass spectrometry, as previously described [21, 22]. In the present study, we only analyzed 36 metabolites previously shown to be associated with BMI [23], including 1,5-anhydroglucitol, 1-oleoylglycerophosphocholine (18:1), 2-hyd roxybutyrate, 2-linoleoylglycerophosphocholine, 3-(4-hyd roxyphenyl)lactate, 3-hydroxyisobutyrate, 3-methyl-2-oxobutyrate, 3-methyl-2-oxovalerate, 4-methyl-2-oxopentanoate, 7-alpha-hydroxy-3-oxo-4-cholestenoate, alpha-hydro xyisovalerate, andro steroid monosulfate 2, asparagine, benzoate, butyrylcarnitine, carnitine, gamma-glutamyli soleucine, gamma-glutamyltyrosine, glutamate, glycerol, glycine, hexanoylcarnitine, histidine, isoleucine, isovalerylcarnitine, kynurenine, lactate, lathosterol, leucine, mannose, N-acetylglycine, palmitoyl sphingomyelin, phenyla lanine, propionylcarnitine, tyrosine, and valine. These metabolites presented with a missing value rate of less than $5 \%$. The missing values were imputed with the smallest detected value. We were unable to analyze the BMIassociated metabolite 1-eicosadienoylglycerophosphochol ine [23], as it was undetectable in the present study cohort.

\section{Statistical analyses \\ Ethnic characterization}

Ethnic characterization of each participant was assessed using the iSelect Metabochip DNA microarrays (Illumina), as previously described [24].

\section{Association analyses between AMY1/AMY2 plasma enzymatic activity or AMY1A/AMY2A copy number and metabolic traits in D.E.S.I.R}

The associations between metabolic quantitative traits (except BMI) and enzymatic activity of AMY1/AMY2 or $A M Y 1 A / A M Y 2 A$ copy number were assessed through linear regression models adjusted for age, sex, BMI, daily alcohol consumption, smoking status, and the first two principal components for ethnicity as previously described [24]. We used the same models for the analysis of plasma metabolites, with the same adjustments (including or not BMI). The analysis of BMI was adjusted for age, sex, daily alcohol consumption, smoking status, and the first two principal components for ethnicity.

The effect of AMY1 or AMY2 activity at baseline on the change in BMI during the 9-year follow-up was assessed through a linear regression model adjusted for age at baseline, sex, BMI at baseline, daily alcohol consumption, smoking status, and the first two principal components for ethnicity. The effect of BMI at baseline on the change in AMY1 or AMY2 activity during the 9-year follow-up was assessed through a linear regression model adjusted for age at baseline, sex, AMY1 or AMY2 activity at baseline, daily alcohol consumption, smoking status, and the first two principal components for ethnicity.

Of note, BMI, aspartate aminotransferase, fasting insulin, triglyceride levels, and the homeostasis model assessment of beta-cell function (HOMA-2B) and of insulin resistance (HOMA-2IR) were logarithmically transformed before statistical analysis. 
Association analyses of glucose-related traits were performed in non-diabetic individuals only. Association analyses of lipid traits were performed in participants who did not use any lipid-lowering drugs at baseline. Association analyses of blood pressure were performed in participants who did not use any drugs against hypertension at baseline.

HOMA-2B and HOMA-2IR were calculated in D.E.S.I.R. participants as previously described [24]. In each regression model, traits were analyzed as dependent variables whilst copy number and enzymatic activities were used as covariates.

\section{Mendelian randomization analysis between $B M I$ and $A M Y 1 /$ AMY2 plasma enzymatic activity}

The causal effect between BMI and AMY1 or AMY2 plasma enzymatic activity was estimated using a Mendelian randomization approach $[25,26]$.

BMI $\rightarrow$ AMY1/AMY2 We used single nucleotide polymorphisms (SNPs) previously found to be genome-wide significantly associated with BMI [27] as genetic instruments for this analysis. We excluded 14 SNPs with known pleiotropic effects on non-anthropometric traits (Additional file 3). Among the remaining $83 \mathrm{SNPs}$, four were not testable through the Illumina Metabochip DNA microarray (rs12016871 within MTIF3 locus, rs16851483 within RASA2 locus, rs17001654 within SCARB2 locus, and rs9641123 within CALCR locus) and one SNP did not pass the quality control (rs12566985 within the FPGT locus). These five SNPs were replaced with proxies $\left(R^{2} \geq 0.64\right.$; Additional file 3). For each of the 83 instrumental genetic variables, we estimated causal effects of BMI on AMY1 or AMY2 plasma enzymatic activity as ratios between the SNP effect sizes on plasma AMY1 or AMY2 enzymatic activity (measured in D.E.S.I.R.) over the SNP effect size on BMI (obtained from Locke et al. [27]). Standard errors for these causal estimates were derived by replacing in the former calculations each SNP effect size on AMY1 or AMY2 plasma enzymatic activity with its corresponding standard error estimated within D.E.S.I.R. The 83 values of causal effects of BMI on AMY1 or AMY2 plasma enzymatic activity were collapsed into single estimates (one for each enzymatic activity) using inverse-variance weighting [25]. Since no published genome-wide association studies on amylase activities were available, we used as an alternative approach, the two-stage least-squares (TSLS) regression to estimate the causal effect of BMI on AMY1 or AMY2 plasma enzymatic activity using D.E.S.I.R. data. This analysis used as the instrumental variable the genetic risk score, calculated as the sum of alleles increasing BMI over the 83 selected SNPs. We did not observe any residual effect of BMI-associated SNPs on amylase activities $(P>0.2)$. To ensure that cryptic pleiotropic effects among the 83 SNPs were not influencing our estimates of causal effect of
BMI on AMY1 and AMY2 plasma enzymatic activities, we used Egger regression to test for the significance of the intercept [28]. We found no significant effect of pleiotropy $(P=0.41$ for AMY1 activity, and $P=0.49$ for AMY2 activity).

AMY1/AMY2 $\rightarrow$ BMI We were unable to use $A M Y 1 A$ or $A M Y 2 A$ copy number as instrumental variables to assess the inverse causation between AMY1 or AMY2 plasma enzymatic activity and BMI as they both showed a residual association with BMI after adjusting for the corresponding plasma enzymatic activity $(P<0.001$; Additional file 4$)$. We therefore looked for other instruments by testing the association between SNP genotyped on the Metabochip DNA microarray (Illumina) and AMY1 or AMY2 plasma enzymatic activity in the D.E.S.I.R. participants. This association was assessed using linear regression of AMY1 or AMY2 plasma enzymatic activity on genotyped SNP adjusted for age, sex, BMI, and the first two principal components for ethnicity. Subsequently, the significant associations between SNPs and AMY1 or AMY2 plasma enzymatic activity (after Bonferroni correction: $P<4 \times 10^{-7}=0.05 \div 124,571$ tested SNPs) were confirmed in ABOS. The combined analyses were performed using a weighted inverse normal method via the function "metagen", with a fixed effect, in the "META" R package. No heterogeneity was observed $(P>0.05)$. A good instrument was consequently defined as a SNP significantly associated $\left(P<4 \times 10^{-7}\right)$ with AMY1 or AMY2 plasma enzymatic activity, without showing any residual association with BMI $(P>0.05)$. Given these instruments, the causal effect of AMY1 or AMY2 plasma enzymatic activity on BMI was estimated using TSLS regression as implemented in the $\mathrm{R}$ package ivpack ( $\mathrm{R}$ function ivreg).

\section{Association analyses between AMY1A copy number and obesity risk}

The association between obesity and $A M Y 1 A$ copy number was assessed by a logistic regression model adjusted for age and sex in the two case-control studies. The combined analysis was performed using a weighted inverse normal method via the function "metagen", with a fixed effect, in the "META" R package. No heterogeneity was observed for this combined analysis $(P=0.14)$.

All genetic analyses were performed under an additive model. All statistical analyses were performed using IBM SPSS (version 14.0) or R (version 3.0).

\section{Results}

Association study between plasma enzymatic activity of AMY1 or AMY2 and metabolic traits in D.E.S.I.R.

After adjustment for clinical and ethnic confounders, a significant association was found between plasma enzymatic activity of AMY1 or AMY2 and lower BMI in the French D.E.S.I.R. participants $(\beta=-0.0013 \pm 0.0002 \mathrm{~kg} /$ 
$\mathrm{m}^{2}, P=2.4 \times 10^{-13} ; \beta=-0.0024 \pm 0.0003 \mathrm{~kg} / \mathrm{m}^{2}, P=2.4 \times$ $10^{-13}$, respectively; Table 1$)$. Importantly, the effects of AMY1 and AMY2 activities seemed partly independent as they remained significant when both AMY1 and AMY2 activities were added in the same regression model $\left(\beta=-0.0012 \pm 0.0002 \mathrm{~kg} / \mathrm{m}^{2}, P=2.8 \times 10^{-12} ; \beta\right.$ $=-0.0021 \pm 0.0003 \mathrm{~kg} / \mathrm{m}^{2}, P=4.2 \times 10^{-12}$, respectively).

Furthermore, in non-diabetic participants, we identified a significant association between AMY1 or AMY2 activity and lower fasting plasma glucose levels $(\beta=-0.0019 \pm$ $0.0006 \mathrm{mmol} / \mathrm{L}, P=1.4 \times 10^{-3} ; \beta=-0.0026 \pm 0.0010 \mathrm{mmol} /$ $\mathrm{L}, P=9.2 \times 10^{-3}$, respectively; Table 1 ), as well as higher beta-cell function modeled by HOMA-2B $(\beta=0.0010 \pm$ $0.0004, P=9.0 \times 10^{-3} ; \quad \beta=0.0017 \pm 0.0006, \quad P=4.6 \times 10^{-3}$, respectively; Table 1$)$. These associations remained significant after the inclusion of AMY1 and AMY2 activities in the same regression model $(P<0.05)$. We also found that AMY2 activity was associated with higher fasting serum insulin levels $\left(\beta=0.0025 \pm 0.0009 \mathrm{pmol} / \mathrm{L}, \quad P=7.5 \times 10^{-3}\right.$; Table 1). However, neither AMY2 activity nor AMY1 activity were found to be associated with insulin resistance (modeled by HOMA-2IR; $P>0.05$; Table 1 ).

Regarding lipid traits, we found that AMY1 activity was associated with higher high-density lipoprotein cholesterol levels $(\beta=0.0015 \pm 0.0005 \mathrm{mmol} / \mathrm{L}, P=2.1 \times 10$ ${ }^{-3}$; Table 1$)$, as well as higher apolipoprotein A1 levels $(\beta=0.0008 \pm 0.0003 \mathrm{~g} / \mathrm{L}, P=0.016$; Table 1$)$.
Furthermore, we identified a significant association between AMY1 activity and higher aspartate aminotransferase levels $\left(\beta=0.0017 \pm 0.0004 \mathrm{IU} / \mathrm{I}, P=4.1 \times 10^{-5}\right.$; Table 1$)$, and AMY2 activity was found to be modestly associated with lower systolic blood pressure (SBP; $\beta=-0.067 \pm 0.029 \mathrm{~mm}$ Hg, $P=0.020$; Table 1).

\section{Association study between AMY1A/AMY2A copy number and metabolic traits in D.E.S.I.R.}

When analyzing copy number of $A M Y 1 A$ and $A M Y 2 A$ in D.E.S.I.R. participants through ddPCR (Additional files 5 and 6), we confirmed that even $A M Y 1 A$ copy numbers were more frequent than odd $A M Y 1 A$ copy numbers (Additional files 5 and 6), as shown by Usher et al. [7]. Furthermore, we confirmed that the copy numbers of $A M Y 1 A$ and $A M Y 2 A$ were nearly always both even or both odd (Additional file 6) [7, 8, 10]. Although $A M Y 1 A$ or $A M Y 2 A$ copy number was significantly correlated with AMY1 or AMY2 plasma enzymatic activity, respectively (Spearman test: $\mathrm{R}^{2}=0.34, P<2.2 \times 10^{-16} ; \mathrm{R}^{2}=0.12, P<$ $2.2 \times 10^{-16}$, respectively; Fig. $1 \mathrm{a}$ and $\left.\mathrm{b}\right)$, we only found a nominal association between $A M Y 1 A$ copy number and lower BMI $\left(\beta=-0.0018 \pm 0.0009 \mathrm{~kg} / \mathrm{m}^{2}\right.$ per $A M Y 1 A$ copy, $P=0.044$; Additional file 7). Of note, we found that ddPCRestimated $A M Y 1 A$ copy number is highly correlated with $A M Y 1 A$ copy number previously estimated by qPCR (Spearman test: $\mathrm{R}^{2}=0.86, P<2.2 \times 10^{-16}$; Additional file 8 )

Table 1 Association between plasma enzymatic activity of AMY1 or AMY2 and metabolic traits in D.E.S.I.R.

\begin{tabular}{|c|c|c|c|c|c|c|}
\hline \multirow[t]{2}{*}{ Traits/disorders } & \multicolumn{3}{|l|}{ AMY1 } & \multicolumn{3}{|l|}{ AMY2 } \\
\hline & $\mathrm{n}$ & Effect size $\pm S E^{a}$ & $P$ value & $\mathrm{n}$ & Effect size $\pm S E^{a}$ & $P$ value \\
\hline $\mathrm{BMI}\left(\mathrm{kg} / \mathrm{m}^{2}\right)$ & 3673 & $-0.0013 \pm 0.0002$ & $2.4 \times 10^{-13}$ & 3905 & $-0.0024 \pm 0.0003$ & $2.0 \times 10^{-16}$ \\
\hline $\mathrm{FG}(\mathrm{mmol} / \mathrm{L})$ & 3629 & $-0.0019 \pm 0.0006$ & $1.4 \times 10^{-3}$ & 3860 & $-0.0026 \pm 0.0010$ & $9.2 \times 10^{-3}$ \\
\hline $\mathrm{Fl}$ (pmol/L) & 3625 & $0.0003 \pm 0.0006$ & 0.63 & 3856 & $0.0025 \pm 0.0009$ & $7.5 \times 10^{-3}$ \\
\hline HOMA-2B & 3343 & $0.0010 \pm 0.0004$ & $9.0 \times 10^{-3}$ & 3548 & $0.0017 \pm 0.0006$ & $4.6 \times 10^{-3}$ \\
\hline HOMA-2IR & 3343 & $0.0004 \pm 0.0005$ & 0.40 & 3548 & $0.0007 \pm 0.0008$ & 0.36 \\
\hline $\mathrm{HDL}(\mathrm{mmol} / \mathrm{L})$ & 3432 & $0.0015 \pm 0.0005$ & $2.1 \times 10^{-3}$ & 3637 & $-0.0005 \pm 0.0008$ & 0.58 \\
\hline LDL (mmol/L) & 3427 & $0.0006 \pm 0.0011$ & 0.61 & 3632 & $-0.0006 \pm 0.0019$ & 0.74 \\
\hline $\mathrm{TG}(\mathrm{mmol} / \mathrm{L})$ & 3463 & $0.0003 \pm 0.0006$ & 0.68 & 3673 & $0.0003 \pm 0.0010$ & 0.81 \\
\hline ApoB (g/L) & 3463 & $0.0001 \pm 0.0003$ & 0.83 & 3673 & $-0.0008 \pm 0.0005$ & 0.11 \\
\hline ApoA1 (g/L) & 3463 & $0.0008 \pm 0.0003$ & 0.016 & 3673 & $-0.0009 \pm 0.0005$ & 0.10 \\
\hline $\mathrm{SBP}(\mathrm{mm} \mathrm{Hg})$ & 3245 & $-0.0063 \pm 0.017$ & 0.71 & 3452 & $-0.067 \pm 0.029$ & 0.020 \\
\hline $\mathrm{DBP}(\mathrm{mm} \mathrm{Hg})$ & 3245 & $0.0010 \pm 0.011$ & 0.93 & 3452 & $-0.013 \pm 0.019$ & 0.48 \\
\hline GGT (IU/L) & 3744 & $-0.0025 \pm 0.042$ & 0.95 & 3980 & $0.021 \pm 0.075$ & 0.79 \\
\hline $\mathrm{ALT}(\mathrm{IU} / \mathrm{L})$ & 3741 & $0.032 \pm 0.021$ & 0.13 & 3977 & $-0.025 \pm 0.035$ & 0.47 \\
\hline AST (IU/L) & 3741 & $0.0017 \pm 0.0004$ & $4.1 \times 10^{-5}$ & 3977 & $0.0002 \pm 0.0007$ & 0.76 \\
\hline
\end{tabular}

${ }^{\mathrm{a}}$ Effect size according to adjusted linear regression model. BMI, AST, FI, HOMA-2B, HO MA-2IR, and TG were logarithmically transformed before statistical analysis $A L T$ alanine aminotransferase, $A M Y 1$ salivary amylase, $A M Y 2$ pancreatic amylase, $A p o A 1$ apolipoprotein $A 1, A p o B$ apolipoprotein $B, A S T$ aspartate aminotransferase, $B M I$ body mass index, $C l$ confidence interval, DBP diastolic blood pressure, FG fasting plasma glucose, $F I$ fasting serum insulin, GGT gamma-glutamyl transferase, $H D L$ high-density lipoprotein, $H O M A-2 B$ homeostasis model assessment of beta-cell function, $H O M A-2 I R$ homeostasis model assessment of insulin resistance, $L D L$ low-density lipoprotein cholesterol, SBP systolic blood pressure, SE standard error, TG triglyceride 


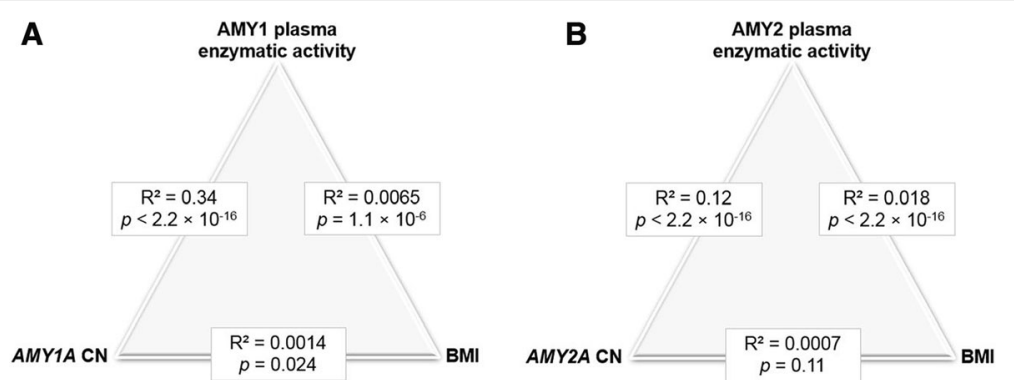

Fig. 1 Correlation analyses between (a) AMY1A copy number, AMY1 activity, and BMI or (b) AMY2A copy number, AMY2 activity, and BMI in D.E.S.I.R. Data are (unadjusted) Spearman correlations with $P$ values

in 2137 participants from D.E.S.I.R. [3]. Furthermore, among these 2137 samples previously assessed, we confirmed a highly significant association between ddPCRestimated $A M Y 1 A$ copy number and lower BMI $(\beta=-$ $0.0043 \pm 0.0011 \mathrm{~kg} / \mathrm{m}^{2}$ per $A M Y 1 A$ copy, $P=1.7 \times 10^{-4}$; Additional files 9 and 10). However, as tackled above, the association between $A M Y 1 A$ copy number and lower BMI was only nominal when we analyzed the whole sample set from D.E.S.I.R. (Additional files 7, 9 and 10).

We did not find other significant associations between $A M Y 1 A$ or $A M Y 2 A$ copy number and metabolic traits $(P>0.05$; Additional file 7$)$.

\section{Assessment of the causal effect between AMY1/AMY2 plasma enzymatic activity and BMI in D.E.S.I.R.}

A Mendelian randomization analysis was subsequently performed to assess causal effects explaining the complex relationship between BMI and AMY1 or AMY2 activity. We found evidence of a causal negative effect of BMI on both AMY1 $\left(\beta_{\text {causal }}=-4.15 \pm 1.66 \mathrm{IU} / \mathrm{L}\right.$ per $\mathrm{kg} / \mathrm{m}^{2}, P=0.012$; Table 2$)$ and AMY2 $\left(\beta_{\text {causal }}=-2.53 \pm 0.94 \mathrm{IU} / \mathrm{L}\right.$ per $\mathrm{kg} / \mathrm{m}^{2}$, $P=7.1 \times 10^{-3}$; Table 2 ) plasma enzymatic activities. These significant effects were confirmed using the TSLS regression (Table 2).

Assessing the inverse relationship (AMY1/AMY2 $\rightarrow$ BMI) turned out to be challenging as we could use neither $A M Y 1 A$ nor $A M Y 2 A$ copy numbers as genetic instruments. Indeed, we found a significant residual association between $A M Y 1 A$ or $A M Y 2 A$ copy number and BMI when adjusting for the corresponding plasma enzymatic activity $(P<0.001$, Additional file 4). As no genome-wide association study for AMY1 or AMY2 plasma enzymatic activity has been performed thus far, we assessed the association between 124,571 SNPs genotyped through the Metabochip DNA microarray and AMY1 or AMY2 activity in D.E.S.I.R. participants, and confirmed the identified associations in another French cohort study (ABOS) in order to find valid genetic instruments. We found one SNP strongly associated with AMY1 activity (PRH1-PRR4 rs10492100: $P=3.3 \times 10^{-11}$; Table 3) and two SNPs strongly associated with AMY2 activity $\left(A M Y 2 B\right.$ rs12075225: $P=2.0 \times 10^{-71} ; A B O$ rs507666:
$P=1.3 \times 10^{-8}$; Table 3$)$. SNP rs12075225 could not be considered as instruments into the Mendelian randomization analysis as we found a residual association between rs12075225 and BMI when adjusting for AMY2 activity $(P<0.05)$. However, we were able to use rs10492100 and rs507666 as instruments to assess the causal effect of AMY1 and AMY2 activities, respectively, on BMI. When using TSLS regression with these instruments, we did not find a significant causal relationship of AMY1 or AMY2 activity on BMI $(P>0.05$; Table 2$)$.

Next, we took advantage of the prospective D.E.S.I.R. study design with measured AMY1 ( $n=679)$ or AMY2 ( $n=$ 705) plasma enzymatic activity after 9 years of follow-up (Additional file 1). Indeed, although confounding can still be present in prospective studies, having consistent results between baseline and follow-up data reinforces the significance of the causal effect estimated besides using Mendelian randomization tools. We found a significant negative effect of BMI at baseline on the change in AMY1 activity $(\beta=-$ $0.20 \pm 0.08 \mathrm{IU} / \mathrm{L}, P=0.014)$ or AMY2 activity $(\beta=-0.18 \pm$ $0.06 \mathrm{IU} / \mathrm{L}, P=3.0 \times 10^{-3}$ ) during the 9-year follow-up, which is in line with the results of the Mendelian randomization analyses showing that BMI negatively impacts amylase activity. Nonetheless, we also identified a significant negative contribution of AMY1 activity at baseline to the change in BMI during the 9-year follow-up $(n=2796 ; \beta=-0.0062 \pm$ $0.0027 \mathrm{~kg} / \mathrm{m}^{2}, P=0.022$ ), which would imply a bidirectional causal negative effect in the relationship between BMI and AMY1 plasma enzymatic activity. The association between AMY2 activity at baseline and the change in BMI during the 9-year follow-up was not significant $(P>0.05)$.

\section{Association study between AMY1A copy number and obesity in adults and children/adolescents}

The uncertainty about the causal effect of lower $A M Y 1 A$ copy number (or AMY1 activity) to higher BMI prompted us to assess the association between $A M Y 1 A$ copy number and obesity risk in two French case-control studies, one including 1179 obese adults and 2220 controls, and the other one including 785 obese children/adolescents and 712 controls (Additional file 2; Additional files 11 and 12). 
Table 2 Estimates of causal relationship between BMI and AMY1 or AMY2 plasma enzymatic activity, using two different methods

\begin{tabular}{|c|c|c|c|c|c|c|}
\hline \multirow{2}{*}{$\begin{array}{l}\text { Relationships } \\
\text { to assess }\end{array}$} & \multicolumn{3}{|c|}{ Inverse variance weighting method } & \multicolumn{3}{|c|}{ Two-stage least squares method } \\
\hline & Effect size \pm SE & $P$ value & Instrument & Effect size \pm SE & $P$ value & Instrument \\
\hline $\begin{array}{l}\mathrm{BMI} \rightarrow \mathrm{AMY1} \\
(\mathrm{n}=3673)\end{array}$ & $-4.15 \pm 1.66$ & 0.012 & $\begin{array}{l}\text { GRS based on } \\
83 \text { SNPs }\end{array}$ & $-0.97 \pm 0.49$ & 0.047 & $\begin{array}{l}\text { GRS based on } \\
83 \text { SNPs }\end{array}$ \\
\hline $\begin{array}{l}\mathrm{BMI} \rightarrow \mathrm{AMY} 2 \\
(\mathrm{n}=3905)\end{array}$ & $-2.53 \pm 0.94$ & $7.1 \times 10^{-3}$ & $\begin{array}{l}\text { GRS based on } \\
83 \text { SNPs }\end{array}$ & $-0.75 \pm 0.28$ & $6.1 \times 10^{-3}$ & $\begin{array}{l}\text { GRS based on } \\
83 \text { SNPs }\end{array}$ \\
\hline $\begin{array}{l}\text { AMY1 } \rightarrow \text { BMI } \\
(n=3673)\end{array}$ & $N A^{a}$ & $N A^{a}$ & $N A^{a}$ & $0.013 \pm 0.046$ & 0.78 & $\begin{array}{l}\text { rs10492100 } \\
\text { (PRH1-PRR4) }\end{array}$ \\
\hline $\begin{array}{l}\mathrm{AMY} 2 \rightarrow \mathrm{BMI} \\
(\mathrm{n}=3905)\end{array}$ & $N A^{a}$ & $N A^{a}$ & $N A^{a}$ & $0.041 \pm 0.097$ & 0.67 & $\begin{array}{l}\text { rs507666 } \\
(A B O)\end{array}$ \\
\hline
\end{tabular}

${ }^{a}$ The inverse variance weighting scheme could not be properly applied to estimate the causal effect of AMY1 or AMY2 plasma enzymatic activity on BMI as to our knowledge, no data on association between SNPs and those activities have been published before

AMY1 salivary amylase, AMY2 pancreatic amylase, BMI body mass index, GRS genetic risk score, NA not applicable, SE standard error, SNP single nucleotide polymorphism

In the French adults, we found that the mean number of $A M Y 1 A$ copies was lower in obese subjects $(6.8 \pm 2.5$; Table 4) than in controls ( $7.0 \pm 2.6$; Table 4$)$, although this difference was not significant when we adjusted the logistic regression model for both age and sex $(P=0.13$; Table 4). In contrast, in the French children/adolescents, we found a significant association between $A M Y 1 A$ copy number and lower obesity risk (odds ratio (OR) per estimated copy 0.94 ; 95\% confidence interval (CI), 0.90$0.98 ; P=7.1 \times 10^{-3}$; Table 4). When we combined the two case-control studies in adults and youths, we identified a significant contribution of $A M Y 1 A$ copy number to lower obesity risk (OR per estimated copy 0.97 ; $95 \%$ CI, 0.94$0.99 ; P=6.8 \times 10^{-3}$; heterogeneity: $P=0.14$; Table 4 ).

\section{Association analysis between known BMI-associated plasma metabolites and AMY1/AMY2 plasma enzymatic activity in D.E.S.I.R.}

Finally, we aimed to assess the association between 36 plasma metabolites known to be associated with BMI [23] and AMY1 or AMY2 plasma enzymatic activity in 718 D.E.S.I.R. participants. First, we confirmed a significant association between these metabolites and BMI in these participants, except for palmitoyl sphingomyelin, which was found to be only metabolite nominally associated with $\mathrm{BMI}(P=0.07)$, although with the same published effect size direction $(\beta<0)$ [23]. Then, we identified significant associations between several metabolites, including branched-chain amino acids (isoleucine, isovalerylcarnitine, and leucine), and AMY1 and/or AMY2 plasma enzymatic activity (Additional file 13), with an effect size direction opposite to the one of BMI effect on the same metabolites (Additional file 13). Interestingly, lactate was significantly associated with higher AMY1 activity, when the regression model was adjusted or not for BMI ( $\beta=0.050 \pm 0.020, P=5.8 \times 10^{-3}$; BMI-adjusted: $\beta=$ $0.058 \pm 0.020, P=1.6 \times 10^{-3}$; Additional file 13).

\section{Discussion}

In the present study, we found that plasma enzymatic activities of both AMY1 and AMY2 were markedly associated with lower BMI and some other related metabolic traits, including lower fasting plasma glucose levels, higher pancreatic beta-cell function, and better lipid profiles, linking starch hydrolysis and metabolism in humans. Although AMY1 or AMY2 plasma enzymatic activity was significantly correlated with the number of copies of $A M Y 1 A$ or $A M Y 2 A$,

Table 3 SNPs found to be significantly associated with AMY1 or AMY2 plasma enzymatic activity in D.E.S.I.R. participants, with a replication study in $\mathrm{ABOS}$ participants

\begin{tabular}{|c|c|c|c|c|c|c|c|c|c|}
\hline & SNP ID & $\begin{array}{l}\text { Chr: position } \\
\text { (Build 37) }\end{array}$ & Closest gene & $\begin{array}{l}\text { Tested allele/ } \\
\text { Other allele }\end{array}$ & Cohort study & $n$ & MAF & $\begin{array}{l}\text { Effect size } \pm \\
\text { SE per allele }\end{array}$ & $P$ value \\
\hline \multirow[t]{3}{*}{$\overline{A M Y 1}$} & rs10492100 & 12:11085820 & PRH1-PRR4 & $\mathrm{C} / \mathrm{T}$ & Discovery & 3673 & 0.21 & $-2.36 \pm 0.39$ & $6.6 \times 10^{-10}$ \\
\hline & & & & & Replication & 488 & 0.25 & $-2.31 \pm 0.85$ & $6.9 \times 10^{-3}$ \\
\hline & & & & & Meta-analysis & 4227 & - & $-2.35 \pm 0.35$ & $3.3 \times 10^{-11}$ \\
\hline \multirow[t]{3}{*}{ AMY2 } & rs12075225 & 1:104115017 & $A M Y 2 B$ & $A / C$ & Discovery & 3905 & 0.10 & $5.11 \pm 0.29$ & $3.3 \times 10^{-70}$ \\
\hline & & & & & Replication & 488 & 0.07 & $3.48 \pm 1.04$ & $9.1 \times 10^{-4}$ \\
\hline & & & & & Meta-analysis & 4227 & - & $4.99 \pm 0.28$ & $2.0 \times 10^{-71}$ \\
\hline \multirow[t]{3}{*}{ AMY2 } & rs507666 & 9:136149399 & $A B O$ & $A / G$ & Discovery & 3905 & 0.22 & $-1.17 \pm 0.22$ & $5.7 \times 10^{-8}$ \\
\hline & & & & & Replication & 488 & 0.21 & $-1.21 \pm 0.60$ & $4.4 \times 10^{-2}$ \\
\hline & & & & & Meta-analysis & 4227 & - & $-1.17 \pm 0.21$ & $1.3 \times 10^{-8}$ \\
\hline
\end{tabular}


Table 4 Association between AMY1A copy number and obesity risk

\begin{tabular}{|c|c|c|c|c|c|c|}
\hline \multirow[t]{2}{*}{ Study } & \multicolumn{2}{|c|}{ Obese cases } & \multicolumn{2}{|c|}{ Controls } & \multirow[t]{2}{*}{ OR $(95 \% C l)^{b}$} & \multirow[t]{2}{*}{$P$ value } \\
\hline & $\bar{n}$ & AMY1A copies ${ }^{a}$ & $\bar{n}$ & AMY1A copies $^{a}$ & & \\
\hline Adults & 1179 & $6.8 \pm 2.5$ & 2220 & $7.0 \pm 2.6$ & $0.98(0.95-1.01)$ & 0.13 \\
\hline Children/Adolescents & 785 & $7.0 \pm 2.6$ & 712 & $7.3 \pm 2.7$ & $0.94(0.90-0.98)$ & $7.1 \times 10^{-3}$ \\
\hline Combined analysis & - & - & - & - & $0.97(0.94-0.99)$ & $6.8 \times 10^{-3}$ \\
\hline
\end{tabular}

${ }^{\text {a }}$ Data are means \pm standard deviation

${ }^{\mathrm{b}}$ Odds ratio per $A M Y 1 A$ copy from a logistic regression adjusted for age and sex $A M Y 1 A$ salivary amylase gene, $C l$ confidence interval, $O R$ odds ratio

respectively (Fig. 1a and b), we found a nominal association between AMY1A copy number and lower BMI in middleaged French adults. However, we identified a significant association between $A M Y 1 A$ copy number and lower risk of obesity in French children, which is in line with our previous study performed in Mexican children [9].

The present study also assessed the hypothesis that pancreatic amylase genes (instead of salivary amylase gene) could actually drive the association with BMI [10]. However, we did not find any significant marginal association between $A M Y 2 A$ copy number and BMI, which makes unlikely a major role for pancreatic amylase gene. Yet, we only genotyped $A M Y 2 A$ copy number and not $A M Y 2 B$ copy number, which is a limitation of our study, even if Usher et al. [7] showed that $A M Y 2 A$ copy number was similar to $A M Y 2 B$ copy number in approximately $95 \%$ of haploid genotypes.

Through a Mendelian randomization analysis, we identified a causal negative effect of BMI on plasma enzymatic activities of both AMY1 and AMY2. In contrast, we failed to find any causal effect of AMY1 or AMY2 plasma enzymatic activity on BMI, although this specific analysis likely lacked sufficient statistical power. Indeed, since we could use neither $A M Y 1 A$ nor $A M Y 2 A$ copy number as a genetic instrument for the analysis, we were deprived of the possibility to utilize some of the strongest potential instruments available. Despite this limitation, we were able to find surrogate instruments that were, however, poorly associated with plasma enzymatic activities of AMY1 and AMY2 compared to their corresponding gene copy number. In addition, since no large genomewide association study for AMY1 or AMY2 plasma enzymatic activity has been performed so far, we were left with a very limited number of useable instruments. The prospective data available in D.E.S.I.R. further supported the negative effect of BMI on AMY1 and AMY2 activities. However, we also found a significant negative contribution of AMY1 activity at baseline to the change in BMI during the 9 years of follow-up, which implies a possibly causative impact of AMY1 activity on decreased adiposity. This was supported by the present results obtained from our obesity case-control study, showing a significant contribution of $A M Y 1 A$ copy number to decreased obesity risk in French children.

The impact of AMY1 activity and AMY1A copy number on adiposity is therefore complex and it seems to interact with the metabolic effect of complex carbohydrate digestion by the gut microflora. Our recent independent digital PCR analyses of AMY1A copy number in Mexican children [9] and our present results in French youths found strong evidence that high $A M Y 1 A$ copy numbers protect against childhood obesity in this high-starch diet populations. In contrast, in French middle-aged adults from the general population, we failed to reproduce these findings. The difference between adults and children may be due to the fact that the heritability of BMI is higher in childhood than in adulthood [29, 30], optimizing the identification of significant associations between genetic events and obesity risk. Furthermore, this difference may be due to different gene-environment interactions depending on age [29,30]. For instance, youths may eat more carbohydrates than adults (as the energy requirements of youths have been shown to parallel their growth rate) [31]. In rodents, it was shown that the SNP at Amy1 locus strongly predicts weight gain after 8 weeks on a highfat, high-sucrose diet, with an associated enrichment in gut bacteria observed in obesity states, which may mediate the metabolic effect of Amy1 expression variation [15].

In D.E.S.I.R., we found that AMY1 plasma enzymatic activity was significantly associated with higher plasma lactate levels independently of BMI. Lactate is a well-known product of complex carbohydrate fermentation by the gut microbiota [32]. It has been proposed that decrease in the lactate/ butyrate ratio can generate an extra 20 calories/day, which may lead to an extra kilogram for weight over a year [32]. Therefore, we suggest that amylase activity, which is associated with higher lactate production, may protect against obesity, especially in individuals with a high-starch diet.

\section{Conclusions}

In conclusion, our systems biology study performed in a prospectively followed population-based European cohort suggests a bidirectional relationship between AMY1 plasma enzymatic activity and adiposity. Altogether, low AMY1 activity due to both genetic and environmental events may modulate human colonic microbiota fermentation of oligosaccharides into short-chain fatty acids via lactate regulation [32], which may have a negative impact on energy harvest, and therefore may aggravate obesity. Further studies are warranted to assess the validity of this hypothesis that, if confirmed, may have clinical implications in obesity treatment [33]. 


\section{Additional files}

Additional file 1: D.E.S.I.R. participants included in the study at baseline and after 9 years of follow-up. (DOC $203 \mathrm{~kb}$ )

Additional file 2: Clinical characteristics of participants included in the two obesity case-control studies. (DOC $30 \mathrm{~kb}$ )

Additional file 3: Effect of SNPs previously found to be associated with BMl on BMl variation in D.E.S.I.R. (DOC $94 \mathrm{~kb}$ )

Additional file 4: Association between copy number of AMY1A or $A M Y 2 A$ and BMI in D.E.S.I.R., respectively adjusted for plasma enzymatic activity of AMY1 or AMY2. (DOC $28 \mathrm{~kb}$ )

Additional file 5: Copy number distributions of (A) AMY1A and (B) AMY2A in D.E.S.I.R. (DOC $185 \mathrm{~kb}$ )

Additional file 6: Unrounded copy number estimates for AMY1A and AMY2A in D.E.S.I.R. (DOC $206 \mathrm{~kb}$ )

Additional file 7: Association between copy number of AMY1A or AMY2A and metabolic traits in D.E.S.I.R. (DOC $46 \mathrm{~kb})$

Additional file 8: Correlation between AMY1A copy number estimated by qPCR versus ddPCR. (DOC $700 \mathrm{~kb}$ )

Additional file 9: Association between ddPCR-estimated AMY1A copy number and BMI in several sets of D.E.S.I.R.: (i) the first set of 2137 samples previously analyzed by qPCR in Falchi et al. [3] paper; (ii) the second set of "all samples minus those 2137 samples", and (iii) all samples from D.E.S.I.R. (DOC $31 \mathrm{~kb})$

Additional file 10: $\mathrm{BMI}$ (means \pm s.e.m.) according to ddPCR-estimated AMY1A copy number in several sets of D.E.S.I.R.: (i) the first set of 2137 samples previously analyzed by qPCR in Falchi et al. [3] paper; (ii) the second set of "all samples minus those 2137 samples (=1463 samples)", and (iii) all samples from D.E.S.I.R. (DOC $187 \mathrm{~kb}$ )

Additional file 11: Distribution of AMY1A copy number in (A) 1179 obese adults and 2220 controls; (B) 785 obese children/adolescents and 712 controls. (DOC $59 \mathrm{~kb}$ )

Additional file 12: Unrounded AMY1A copy number estimates in (A) 1179 obese adults and 2220 controls; (B) 785 obese children/adolescents and 712 controls. (DOC $154 \mathrm{~kb}$ )

Additional file 13: Significant associations between BMl-associated plasma metabolites and AMY1 or AMY2 plasma enzymatic activity in 718 participants from D.E.S.I.R. (DOC $47 \mathrm{~kb})$

\section{Abbreviations}

AMY1: salivary amylase; AMY1A: salivary amylase gene; AMY2: pancreatic amylase; AMY2A: pancreatic amylase gene; BMI: body mass index; ddPCR: droplet digital PCR; HOMA-2B: homeostasis model assessment of beta-cell function; HOMA-2IR: homeostasis model assessment of insulin resistance; qPCR: quantitative RT-PCR; SNP: single nucleotide polymorphism; TSLS: two-stage least-squares.

\section{Acknowledgments}

We are grateful to all participants involved in the genetic study. We thank Tim Spector (King's College London, London, UK) for fruitful discussions. The D.E.S.I.R. Study Group is composed of Inserm-U1018 (Paris: B. Balkau, P. Ducimetière, E. Eschwège), Inserm-U367 (Paris: F. Alhenc-Gelas), CHU d'Angers (A. Girault), Bichat Hospital (Paris: F. Fumeron, M. Marre, R. Roussel), CHU de Rennes (F. Bonnet), CNRS UMR-8199 (Lille: A. Bonnefond, P. Froguel), Medical Examination Services (Alençon, Angers, Blois, Caen, Chartres, Chateauroux, Cholet, LeMans, Orléans and Tours), Research Institute for General Medicine (J. Cogneau), the general practitioners of the region and the Cross-Regional Institute for Health (C. Born, E. Caces, M. Cailleau, N. Copin, J.G. Moreau, F. Rakotozafy, J. Tichet, S. Vol).

\section{Funding}

This work was supported by grants from the French National Research Agency (ANR-10-LABX-46 [European Genomics Institute for Diabetes] and ANR-10-EQPX-07-01 [LIGAN-PM], to PF), from the European Research Council (ERC GEPIDIAB - 294785, to PF), from FEDER, from Qatar Foundation, and from the 'Région Nord Pas-de-Calais'. AB was supported by Inserm. The D.E.S.I.R. study was supported by Inserm contracts with CNAMTS, Lilly, Novartis Pharma, and Sanofi-Aventis, and by Inserm (Réseaux en Santé
Publique, Interactions entre les déterminants de la santé, Cohortes Santé TGIR 2008), the Association Diabète Risque Vasculaire, the Fédération Française de Cardiologie, La Fondation de France, Société Francophone du Diabète, ONIVINS, Ardix Medical, Bayer Diagnostics, Becton Dickinson, Cardionics, Merck Santé, Novo Nordisk, Pierre Fabre, Roche, and Topcon.

\section{Availability of data and materials}

Clinical, metabolic, and genetic data are available according to the rules of each individual cohort and can be requested from the D.E.S.I.R. cohort (Kremlin Bicêtre, France), the ABOS study (Lille, France), the CNRS UMR8199 unit (Lille, France), the Haguenau study (Paris, France), and the Fleurbaix-Laventie Ville Santé study (Lille, France).

\section{Authors' contributions}

Writing - Original Draft, AB, LY, PF; Writing - Review \& Editing, BB, FP, TB; Investigation: $A D, M C a s, E R$, FA; Formal Analysis, $A B, L Y, M C a n ;$ Resources, $R C$, VR, MP, AA, J-MB, CL-M, JW, RR, BB, MM, FP, TB, PF; Conceptualization, AB, LY, PF; Supervision, AB, PF. All authors read and approved the final manuscript.

\section{Competing interests}

The authors declare that they have no competing interests.

\section{Consent for publication}

Not applicable.

\section{Ethics approval and consent to participate}

Ethical approval was obtained from each of the following committees: Bicêtre Hospital (Kremlin Bicêtre, France), Comité Consultatif de Protection des Personnes se prêtant à des Recherches Biomédicales (CPPRB) of Lille - Lille Hospital (Lille, France), and Paris St Louis University (Paris, France). Informed consent was obtained from each subject before participation in the study, in accordance with the Declaration of Helsinki principles. For children or adolescents younger than 18 years, oral consent was obtained, and parents provided written informed consent.

\section{Author details}

'University of Lille, CNRS, Institut Pasteur de Lille, UMR 8199 - EGID, Lille 59000, France. ${ }^{2}$ Institute for Molecular Bioscience, The University of Queensland, Brisbane 4067, Australia. ${ }^{3}$ University of Lille, Inserm, U1190 EGID, Lille 59000, France. ${ }^{4}$ Endocrine Surgery Department, CHU of Lille, Lille 59000 , France. ${ }^{5}$ Qatar Biomedical Research Institute, Qatar Foundation, Doha 5825, Qatar. ${ }^{6}$ Fleurbaix-Laventie Association, Laventie 62840, France. ${ }^{7}$ Inserm CIE 05 - Department of Clinical Epidemiology, Robert Debré Hospital, Paris 75019, France. ${ }^{8}$ Pediatric Endocrine Department, CHU of Lille, Lille 59000, France. ${ }^{9}$ Inserm, U1138, Centre de Recherche des Cordeliers, Paris 75006, France. ${ }^{10}$ Paris-Diderot University, Sorbonne Paris-Cité, Paris 75013, France. ${ }^{11}$ Department of Endocrinology-Diabetology and Nutrition, DHU-FIRE, Bichat Hospital, Assistance Publique-Hôpitaux de Paris, Paris 75018, France. ${ }^{12}$ Inserm, U1018, CESP, Team 5 (EpReC, Renal and cardiovascular Epidemiology), UVSQ-UPS, Villejuif 94807, France. ${ }^{13}$ UF8832 - Biochimie Automatisée, Pôle de Biologie Pathologie Génétique, CHU of Lille, Lille 59000, France.

${ }^{14}$ Department of Genomics of Common Disease, School of Public Health Imperial College London, Hammersmith Hospital, London W12 ONN, UK.

Received: 3 October 2016 Accepted: 9 January 2017

Published online: 23 February 2017

\section{References}

1. Mifflin TE, Hortin G, Bruns DE. Electrophoretic assays of amylase isoenzymes and isoforms. Clin Lab Med. 1986;6:583-99.

2. Perry GH, Dominy NJ, Claw KG, Lee AS, Fiegler H, Redon R, et al. Diet and the evolution of human amylase gene copy number variation. Nat Genet. 2007:39:1256-60.

3. Falchi M, El-Sayed Moustafa JS, Takousis P, Pesce F, Bonnefond A, Andersson-Assarsson JC, et al. Low copy number of the salivary amylase gene predisposes to obesity. Nat Genet. 2014:46:492-7.

4. Viljakainen $\mathrm{H}$, Andersson-Assarsson JC, Armenio M, Pekkinen M, Pettersson $M$, Valta $\mathrm{H}$, et al. Low copy number of the AMY1 locus is associated with early-onset female obesity in Finland. PLoS One. 2015;10, e0131883. 
5. Marcovecchio ML, Florio R, Verginelli F, De Lellis L, Capelli C, Verzilli D, et al. Low AMY1 gene copy number is associated with increased body mass index in prepubertal boys. PLoS One. 2016;11, e0154961.

6. Choi Y-J, Nam Y-S, Yun JM, Park JH, Cho BL, Son H-Y, et al. Association between salivary amylase (AMY1) gene copy numbers and insulin resistance in asymptomatic Korean men. Diabet Med J Br Diabet Assoc. 2015;32:1588-95.

7. Usher CL, Handsaker RE, Esko T, Tuke MA, Weedon MN, Hastie AR, et al. Structural forms of the human amylase locus and their relationships to SNPs, haplotypes and obesity. Nat Genet. 2015;47:921-5.

8. Yong RYY, Mustaffa SB, Wasan PS, Sheng L, Marshall CR, Scherer SW, et al. Complex copy number variation of AMY1 does not associate with obesity in two East Asian cohorts. Hum Mutat. 2016;37:669-78.

9. Mejía-Benítez MA, Bonnefond A, Yengo L, Huyvaert M, Dechaume A, Peralta-Romero J, et al. Beneficial effect of a high number of copies of salivary amylase AMY1 gene on obesity risk in Mexican children. Diabetologia. 2015;58:290-4.

10. Carpenter D, Dhar S, Mitchell LM, Fu B, Tyson J, Shwan NAA, et al. Obesity, starch digestion and amylase: association between copy number variants at human salivary (AMY1) and pancreatic (AMY2) amylase genes. Hum Mol Genet. 2015;24:3472-80.

11. Boettger LM, Salem RM, Handsaker RE, Peloso GM, Kathiresan S, Hirschhorn $\mathrm{JN}$, et al. Recurring exon deletions in the HP (haptoglobin) gene contribute to lower blood cholesterol levels. Nat Genet. 2016;48:359-66.

12. White S. Counting copy number and calories. Nat Genet. 2015;47:852-3.

13. Mandel AL, Breslin PAS. High endogenous salivary amylase activity is associated with improved glycemic homeostasis following starch ingestion in adults. J Nutr. 2012;142:853-8.

14. Nakajima K, Nemoto T, Muneyuki T, Kakei M, Fuchigami H, Munakata H. Low serum amylase in association with metabolic syndrome and diabetes: a community-based study. Cardiovasc Diabetol. 2011;10:34.

15. Parks BW, Nam E, Org E, Kostem E, Norheim F, Hui ST, et al. Genetic control of obesity and gut microbiota composition in response to high-fat, highsucrose diet in mice. Cell Metab. 2013;17:141-52.

16. Balkau B, Eschwege E, Tichet J, Marre M. Proposed criteria for the diagnosis of diabetes: evidence from a French epidemiological study (D.E.S.I.R.). Diabetes Metab. 1997;23:428-34.

17. American Diabetes Association. Standards of medical care in diabetes-2014. Diabetes Care. 2014;37 Suppl 1:S14-80.

18. Wolowczuk I, Hennart B, Leloire A, Bessede A, Soichot M, Taront S, et al. Tryptophan metabolism activation by indoleamine 2,3-dioxygenase in adipose tissue of obese women: an attempt to maintain immune homeostasis and vascular tone. Am J Physiol Regul Integr Comp Physiol. 2012;303:R135-43.

19. Leger J, Levy-Marchal C, Bloch J, Pinet A, Chevenne D, Porquet D, et al. Reduced final height and indications for insulin resistance in 20 year olds born small for gestational age: regional cohort study. BMJ. 1997;315:341-7.

20. Romon M, Lafay L, Bresson JL, Oppert J-M, Borys J-M, Kettaneh A, et al. Relationships between physical activity and plasma leptin levels in healthy children: the Fleurbaix-Laventie Ville Santé II Study. Int J Obes Relat Metab Disord. 2004;28:1227-32.

21. Evans AM, DeHaven CD, Barrett T, Mitchell M, Milgram E. Integrated, nontargeted ultrahigh performance liquid chromatography/electrospray ionization tandem mass spectrometry platform for the identification and relative quantification of the small-molecule complement of biological systems. Anal Chem. 2009;81:6656-67.

22. Cheng J, Joyce A, Yates K, Aouizerat B, Sanyal AJ. Metabolomic profiling to identify predictors of response to vitamin $\mathrm{E}$ for non-alcoholic steatohepatitis (NASH). PLoS One. 2012;7, e44106.

23. Moore SC, Matthews CE, Sampson JN, Stolzenberg-Solomon RZ, Zheng W, Cai $\mathrm{Q}$, et al. Human metabolic correlates of body mass index. Metabolomics. 2014;10:259-69.

24. Vaxillaire M, Yengo L, Lobbens S, Rocheleau G, Eury E, Lantieri O, et al. Type 2 diabetes-related genetic risk scores associated with variations in fasting plasma glucose and development of impaired glucose homeostasis in the prospective DESIR study. Diabetologia. 2014;57:1601-10.

25. Ahmad OS, Morris JA, Mujammami M, Forgetta $V$, Leong $A$, Li R, et al. A Mendelian randomization study of the effect of type-2 diabetes on coronary heart disease. Nat Commun. 2015;6:7060.

26. Fall T, Xie W, Poon W, Yaghootkar H, Mägi R. GENESIS Consortium, et al. Using genetic variants to assess the relationship between circulating lipids and type 2 diabetes. Diabetes. 2015;64:2676-84.
27. Locke AE, Kahali B, Berndt SI, Justice AE, Pers TH, Day FR, et al. Genetic studies of body mass index yield new insights for obesity biology. Nature. 2015;518:197-206

28. Bowden J, Davey Smith G, Burgess S. Mendelian randomization with invalid instruments: effect estimation and bias detection through Egger regression. Int J Epidemiol. 2015;44:512-25.

29. Elks CE, den Hoed M, Zhao JH, Sharp SJ, Wareham NJ, Loos RJF, et al. Variability in the heritability of body mass index: a systematic review and meta-regression. Front Endocrinol. 2012;3:29.

30. Haworth CMA, Carnell S, Meaburn EL, Davis OSP, Plomin R, Wardle J. Increasing heritability of $\mathrm{BMI}$ and stronger associations with the FTO gene over childhood. Obes Silver Spring Md. 2008;16:2663-8.

31. Story M, Stang J. Nutrition Needs of Adolescents. Guidelines for Adolescent Nutrition Services. Chapter 3. 2005; pp. 21-34. http://www.epi.umn.edu/let/ pubs/adol_book.shtm.

32. Aguirre M, Jonkers DMAE, Troost FJ, Roeselers G, Venema K. In vitro characterization of the impact of different substrates on metabolite production, energy extraction and composition of gut microbiota from lean and obese subjects. PLoS One. 2014;9, e113864.

33. Pedersen HK, Gudmundsdottir V, Nielsen HB, Hyotylainen T, Nielsen T, Jensen $\mathrm{BAH}$, et al. Human gut microbes impact host serum metabolome and insulin sensitivity. Nature. 2016:535:376-81.

\section{Submit your next manuscript to BioMed Central and we will help you at every step:}

- We accept pre-submission inquiries

- Our selector tool helps you to find the most relevant journal

- We provide round the clock customer support

- Convenient online submission

- Thorough peer review

- Inclusion in PubMed and all major indexing services

- Maximum visibility for your research

Submit your manuscript at www.biomedcentral.com/submit
CioMed Central 